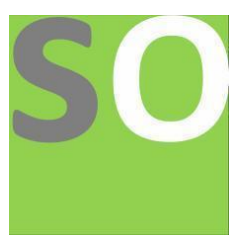

Article title: The Effect of Caffeine on Athletic Performance

Authors: Nabeel Hussain[1]

Affiliations: Saba University School of Medicine, Saba[1]

Orcid ids: 0000-0002-2776-0398[1]

Contact e-mail: hussain.nabeel@outlook.com

License information: This work has been published open access under Creative Commons Attribution License http://creativecommons.org/licenses/by/4.0/, which permits unrestricted use, distribution, and reproduction in any medium, provided the original work is properly cited. Conditions, terms of use and publishing policy can be found at https://www.scienceopen.com/.

Preprint statement: This article is a preprint and has not been peer-reviewed, under consideration and submitted to ScienceOpen Preprints for open peer review.

DOI: 10.14293/S2199-1006.1.SOR-.PPAGBLN.v1

Preprint first posted online: 31 January 2021

Keywords: caffeine, athletic performance, exercise, physical performance, sports 


\title{
The Effect of Caffeine on Athletic Performance
}

\author{
Nabeel Hussain \\ SABA University School of Medicine \\ 2 Napanee Street, Brampton ON, L6S 4X8, Canada \\ (647) 606-7173, n.hussain@saba.edu \\ Advisor: Dr. DeLaCruz
}

Article Word Count: 8280

Hypothesis: The intake of caffeine can increase physical performance during athletic activity 


\begin{abstract}
Hypothesis: The intake of caffeine can increase physical performance during athletic activity Methods: A search for primary sources was done using PubMed with MeSH terms. The search was limited to randomized controlled trials that were published between 2015 and 2020. After application of inclusion and exclusion criteria, seven articles were selected for this literature review.
\end{abstract}

Results: Of the seven randomized controlled trials selected, six demonstrated caffeine ingestion led to a statistically significant increase in physical performance. One of the randomized controlled trials found no statistically significant relationship between caffeine and run timings. The level set for statistical significance for this literature review was set to $\mathrm{p}<0.05$.

Conclusion: With regards to the results of the selected studies, caffeine was shown to have ergogenic activity and was able to increase physical performance during exercise and sporting competition through multiple mechanisms. Further research should be done with greater sample sizes to determine the effect of rate of metabolism on caffeine activity and to compare caffeine responders and non-responders.

Word count: 176

Keywords: Caffeine, athletic performance, exercise, physical performance, sports 


\section{INTRODUCTION}

Caffeine is the most widely utilized psychoactive substance in the world and may be ingested in various forms such as capsules and beverages. Caffeine use is completely unregulated throughout the world and many individuals consume it daily for its stimulant effects on both the central and autonomic nervous system. Caffeine has an ability to induce alertness, increase ability to sustain intellectual activity and enhance body reactivity to stimulus (Vanderveen, 2001). A widely studied benefit of caffeine is its effect on physical performance especially when utilized before athletic activity. Dietary supplements are consumed by individuals at all competitive levels and competitive level rises in a sport, athletes seek any advantage to enhance their performance. In comparison to anabolic steroids and other performance-enhancing drugs, improvements by caffeine are not as exaggerated but may still be greatly beneficial for any type of athlete. Athletic performance is categorized into physical output and the neurocognitive abilities of an athlete. Therefore, enhancing either of these factors can benefit the performance of athletes in a sport. Caffeine emerges as a potential performance enhancer due to its stimulatory effect on the nervous system with direct and hormonal effects on skeletal muscle function. The use of caffeine can easily be implemented due to the Olympic committee and all major sporting organizations have already permitted its use.

The effects of caffeine are carried out through multiple biochemical reactions. Caffeine acts as an adenosine receptor antagonist to decrease the impairment of attentional processes and counteract the consequences sleep deprivation, to improve focus and reduce fatigue (Urry, 2014). Caffeine is a non-selective antagonist to both the A1 and A2 adenosine receptors and indirectly effects the release of other neurotransmitters such dopamine, GABA and acetylcholine. Caffeine has the ability to reduce myocyte glycogen consumption by increasing lipolysis of triglycerides 
which can lead to reduced lactic acid production by working muscles. This effect occurs due to the methylxanthine function of caffeine, which allows it to inhibit phosphodiesterase in order to increases cAMP concentration within skeletal and adipose tissue. Elevated cAMP levels induce release of norepinephrine and epinephrine to stimulates the cardiovascular system leading to increased coronary and skeletal blood flow. As a result, there is enhanced endurance through the reduction of pain and discomfort associated with lactic acid accumulation and lengthening of the time muscle fibers take to fatigue. Caffeine has been shown to reduce exertion perceived by muscles, resulting in increased power output due to muscle failure points being heightened so muscle fibres can output more work over time (Grgic, 2017).

Caffeine is readily absorbed in the digestive tract within an hour of ingestion (Vanderveen, 2001) and redistributes throughout the body by its lipophilic nature. It serves as an ideal performance enhancer because it can be ingested in various forms, is widely available, inexpensive and is relatively safe. The half-life of caffeine ranges from 2.5 to 10 hours allowing its effects to last through the entire competition. Caffeine has been shown to enhance skeletal muscle endurance and strength leading to elevated sprinting speed as well as increasing the speed and number of high-intensity movement performed by the athlete (Puente, 2017). Use of caffeine would likely be most beneficial to sports that require short bursts of explosive movements.

The study of caffeine and its relation to athletic performance is important to many sports. Caffeine has been thought to have a positive relationship to improvements of muscular performance, therefore athletes should be encouraged to utilize caffeine to maximize their probability of succeeding during athletic competition. 


\section{METHODS}

The major source of information for both the results and introduction of this study was PubMed which served as a database providing meta-analyses and systemic reviews for general information and randomized controlled trials for the results. Using Medical Subject Headings $(\mathrm{MeSH})$, a wide-ranging search was conducted on caffeine and its relation to athletic performance using terms, ("Caffeine"[Mesh]) AND "Athletic Performance"[Mesh] which yielded 464 results. To narrow the search further, search term "AND "Exercise"[Mesh]” was added to locate studied using exercises in a quantitative manner to measure the effect of caffeine, which minimized the search to 219 results. The search was limited to studies published within 5 years from the initiation of this literature review, to center the search results on more recent publications. Inclusion criteria for the studies was limited to randomized control trials. Including all inclusion criteria, the search results generated 45 possible articles.

Articles were screened based on their methods and abstract sections to determine if they most clearly displayed the effect caffeine on performance in a sport, sports related exercise, general exercise or sport-specific training techniques. Studies that did not measure dependent variables in any of these forms or included multiple independent variables were excluded. Studies were also excluded if they focused solely on the neurological effects of caffeine in sports. This exclusion was included due to the difficulty in separating caffeine's physiological activity and neuronal actions as well as the inability to remove the confounding bias from muscle memory and timing had on physical performance. Caffeine ingested in energy drink form was excluded to minimize the confounding effects carbohydrates and electrolytes ingestion had on caffeine. Other criteria for exclusion, were if the study parameters were too specific and not reflective of conditions a normal athlete would find themselves in. Studies not included in the 
study were used to provide background information to better understand the results of the chosen studies and provide insight into the multi-faceted influence caffeine on physical performance. After the exclusion process, of seven journal articles were selected to be studied in this paper. A PRIMSA flow diagram in Appendix A was included to demonstrate how papers were excluded and selected.

\section{RESULTS}

The contents of this literature review focused on seven randomized controlled trials that were analyzed to provide a comprehensive view of how caffeine may alter athletic performance. The studies were based on various sports and general exercises to maximize the generalizability of the results (See Table 1 on Appendix B). The exercises used to measure the effects of caffeine could be categorized into maximum performance-based and endurance-based physical activity. Maximum performance exercises consisted of strength-training, explosive movements and speed tests which demonstrated the effects of caffeine on maximal skeletal muscle output during a short time period. Examples of these exercises include one repetition maximum and sprint timings. On the other hand, endurance-based exercises demonstrated the caffeinated effect on sustained skeletal muscle activity and alterations on the cardiopulmonary system. These exercises include long duration runs or output over a full game of a specific sport.

The first article analyzed was performed by Puente et al (2017), to determine if caffeine could improve basketball performance on twenty experienced male and female basketball players. The participants were ideal for the study because they were composed of both female professional basketball players and male semi-professional players with extensive previous physical training and sport-specific experience. Age range of the participants were similar with 
averages for females being 27.9 years and males 27.1 years. All participants had over 10 years of experience playing basketball to reduce confounding from discrepancies in basketball experience. Participants were advised to refrain from caffeine use or use less than 100mg per day during the course of the study. Female participants were only tested during the luteal phase of their menstrual cycle to avoid performance changes that may occur through their ovulatory cycle. The study was designed as a randomized controlled trial, participates ingested either a placebo or $3 \mathrm{mg}$ of caffeine per kilogram of body mass 1 hour before the experimental trial began to provide ample time for caffeine to be absorbed. Performance was tested by ten repetitions of the Abalakov jump, which tests for vertical reach, a change of direction and acceleration test (CODAT) and two free throws. For the CODAT, the first five repetitions were performed without the basketball and the last five were performed with the ball, to measure any differences in sprinting speed with and without dribbling. Following performance testing, two 10-minute stimulated games were recorded and analyzed by two basketball statistical specialists to measure the performance index rating of each individual using FIBA standards, which used the number of movements and actions conducted by a subject through the game. Afterwards participants completed a survey regarding perception of their strength, stamina and overall exertion.

The caffeine group was found to have an increase in the average height reached during the ten repetitions of the Abalakov jump test $(37.3 \pm 6.8$ for placebo vs. $38.2 \pm 7.4 \mathrm{~cm}$ for caffeine; $95 \% \mathrm{CI}=0.3$ to $1.6 \mathrm{~cm})$. In reference to the placebo, participants who had ingested caffeine during the game had an increase to their average jump height, number of free throws, offensive rebounds, total rebounds and quantity of assists. Caffeine was found to have no effect on the accuracy of free throws indicating it did not impact sport-specific accuracy. The increased number of assists in caffeinated individuals $(1.1 \pm 0.9$ vs $2.1 \pm 1.6$ assists $)$ demonstrates 
improvement in player coordination and timing. For the CODAT, a select number of repetitions shows improvement but there was no change in overall average time to completion or maximum running speed between the caffeine and placebo groups. In comparison to the placebo, the caffeine group had increased performance index ratings ( $8.4 \pm 8.3 \mathrm{vs} 11.6 \pm 7.3)$, number of impacts per minute between players (396 \pm 43 vs. $410 \pm 41$ impacts $/ \mathrm{min} ; \mathrm{p}<0.001)$. Overall caffeine was able to act as an ergogenic agent to increase physical and overall performance during basketball but was found to have negative effects on sleeping patterns, with over half of participants reported insomnia. Long term changes to sleep may negatively impacts performance by depriving athletes of adequate rest. Statistical analysis for this study was done with the Shapiro-Wilk test to determine the normality of each variable, followed by a Student's t-test on each of the normally distributed dependent variables to compare the caffeinated group to the placebo. Afterwards for the Abalakov jump and CODAT, the caffeine and placebo groups were compared using a two-way analysis of variance (ANOVA). Statistical significance level was set to $\mathrm{p}<0.05$ and $95 \%$ confidence intervals for the difference of means between the placebo and caffeine groups were determined.

A study conducted by Marques et al. (2018) aimed to investigate whether caffeine consumption could reduce the time needed to complete an 800-meter run in twelve overnight fasted male runners. Twelve individuals were selected for the study as it was found only ten subjects were required to obtain statistically significant results when a pilot study was conducted by the same research group. The study utilized a double-blind format and randomized subject into either the decaffeinated coffee placebo group or 5.5mg per kilogram body mass of caffeine group for the first trial and for the second trial subjects were crossed over to the other group. Both the caffeine and the placebo were dissolved in $200 \mathrm{ml}$ of hot water and participants had 2 
minutes to consume the beverage, this was followed by an hour wait period for caffeine absorptions. Study participants were required to fast overnight for a period of 8 to 10 hours prior to the trial to reduce available levels of carbohydrates and were told to refrain from caffeine for 48 hours. This prevented the long-term effects of caffeine from interfering with the study results. Blood pressure, serum lactate and glucose levels were measured before and after each trial. Following the 8-10 hour fast and consumption of their respective beverages, the participants were required to run two laps around a 400-meter track. Both sets of trials were conducted on the during the morning, with subjects being adequately hydrated and instructed to exert maximal effort. Following the trials, the researchers found serum glucose, serum lactate and systolic blood pressure were elevated almost immediately after the trial began in the caffeine and placebo groups but there was no statistically significant difference between the groups. Diastolic pressure was found to have no changes during the trials compared to baseline in either group. These findings are thought to be independent of caffeine and were exercise-induced physiological reactions. No differences were found in trial timings (Decaffeinated: $2.38+0.10$ vs. Caffeine: $2.39+0.09$ minutes, $p=0.336$ ), and ratings of perceived exertion (Decaffeinated: $16.50+2.68$ vs. Caffeine: $17.00+2.66, \mathrm{p}=0.326$ ) for both groups. Furthermore, the researchers concluded that consumption of caffeine was unable to improve running times for 800 -meter races in fasted runners. Statistical analysis was conducted using ANOVA to determine differences between the caffeine and placebo groups for serum glucose and lactic acid levels, followed by a t-test to determine differences in trial times and rating of perceived exertion. Level of statistical significance was set at $\mathrm{p}<0.05$ for tests conducted.

A double-blind randomized control trial was conducted by Grgic et al. (2017) to investigate the short-term effects of caffeine on athletic performance through changes in 
muscular strength, power and endurance. The study also aimed to determine if perceived exertion and pain perception were impacted by caffeine. Seventeen male participants were selected that had over twelve months of prior resistance-based training with resistance training done atleast three times a week for the last six months prior to the induction of the study. Participants with neuromuscular or musculoskeletal disorders were excluded. Participants were given $6 \mathrm{mg}$ per kilogram body mass of anhydrous caffeine to maximize serum caffeine levels (Graham, 1995) or a placebo. Three study trials were conducted, each separated by a from each other. The first session served as a control and the following two session had subjects randomized into the caffeine or placebo groups. The placebo and caffeine were diluted into 250 $\mathrm{mL}$ of water with $20 \mathrm{~g}$ of orange beverage powder. Following ingestion, participants waited one hour to allow for absorption of the caffeine. Before performing any of the exercises, a timed 5minute stationary bike session was conducted, followed by multiple push-ups and "walk-outs" to serve as warmups. The researchers utilized different exercises to measure power, strength and endurance. To measure muscular power, a vertical jump exercise and seated medicine ball throws were used, whereas for muscular strength, a one repetition maximum for the barbell back squat and bench press exercises were utilized. The following equation 1 repetition maximum $=$ Weight x (36/ (37 - Repetitions) (Bryzcki, 1993) was used in order to calculate participants' one repetition maximum and the same weight was repeated through the sessions. When measuring for changes in muscular endurance, number of repetitions for the barbell back squat and bench press until muscle failure were used by the study group. Muscular power was measured first, followed by strength then muscular endurance with each being measured with two exercise, one for lower and one for upper body. Rating of perceived exertion and pain perception were 
measured using the Borg scale (Borg, 1970) with participants being familiarized with the scales during the first session.

Using an ANOVA analysis, the caffeine group had improvement to the one repetition maximum for the barbell back squat, reduction in ratings of perceived exertion for the back squat and reduced pain perception for the bench press one repetition maximum. The researchers found improvement to the distance the medicine ball was thrown for the caffeine group. Acute enhancements to lower body strength performance were observed with caffeine intake, whereas upper body muscular strength was unaffected by the ingestion of caffeine. A significant reduction in pain perception was found during lower body muscular strength testing. Additionally, upper body muscular power was positively impacted by caffeine intake while lower body muscular power remained unaffected. Some variations in muscular strength and power were observed in individuals of the caffeine group which may suggest differences in motor unit recruitment. There was no difference in rating of perceived exertion observed for the bench press, possibly due to its simpler nature and shorter range of motion. Since the bench press was performed at the end of each sessions, the results for it could be inaccurate due the buildup of fatigue from previous exercises. The results of this study indicate a reduction in perceived pain may allow subjects to perform at a higher level. For muscular endurance, there were no significant differences found for either upper or lower body endurance between the caffeine and placebo groups. Additionally, no improvements to pain perception or rating of perceived exertion were found during muscular endurance testing. Lack of significant changes observed for muscular endurance may be due to fatigue from intensive muscular power and strength testing done earlier in the session. 
Statistical analysis for this study was done using the Shapiro-Wilk test to determine normality of each variable. Afterwards ANOVA was used to determine if there were statistically significant differences in muscular power, strength and endurance between the caffeinated and placebo groups. Level of statistical significance was set to $p<0.05$ and each exercise had a 95\% confidence interval calculated for it using Microsoft Excel.

Cheng et al. (2016) conducted a double-blind, randomized crossover study to investigate the effect of caffeine on the performance of athletes in a 3 minute all out test (3MT) and changes to their plasma electrolytes. Fifteen male college basketball players with an average caffeine intake of 50 to $100 \mathrm{mg}$ per day were selected. Participants were given either $6 \mathrm{mg}$ per kilogram mass of caffeine or a placebo in capsular form, followed by an hour where they laid supine in a dark, quiet room to allow for adequate absorption of caffeine. Following a 5-minute warmup, study subjects conducted the 3MT, which consisted of 3 minutes of baseline peddling on a stationary bike, followed immediately by 3 minutes of all out effort pedaling. Testing was done in 4 session over 3 weeks, with the first session participants completed an incremental cycling test in order to measure their maximal oxygen uptake $\left(\mathrm{VO}_{2}\right.$ max $)$ and gas exchange threshold (GET). The cycling test involved subjects cycling at a constant revolutions per minute on a stationary bike until exhaustion, during which the resistance of the bike was increased by 100W every thirty minutes. Gas exchange threshold was determined during the cycling test, using a face mask attached to a gas analysis system. The second visit, the athletes performed the 3MT to familiarize and train themselves for 3MT. In the third and fourth visit, the trials were spaced one week apart for washout and participants were randomized into either the caffeine group or placebo group. The results of the $3 \mathrm{MT}$ were used to determine critical power and the curvature constant, which have direct associations with aerobic and anaerobic capabilities, 
respectively. Both were measured by exercising until exhaustion and calculation relations between the work done during this period. Critical power represents the upper limit of work that can be done at a constant state through metabolism. Curvature constant is work done past critical power that uses only available energy resources to power the muscles. Researchers believe caffeine was able to prolong the time to exhaustion by increasing both critical power and curvature constant leading to improve overall endurance. Caffeine's benefits are likely to be on observed better in trained athletes due to the subtle enhancement motor unit recruitment and reduction to perceived effort because less confound would arise proper diet, rest and training. Serum potassium was measured because high levels can reduce membrane potential leading to muscle fatigue and decline in force production. The study team believed caffeine could counteract these effects by reducing serum potassium buildup by promoting $\mathrm{Na}^{+} / \mathrm{K}^{+}$ATPase pump activity and shunting potassium back inside muscle cells.

After compiling the results, researchers found subjects in the caffeine group had a higher work end power than the placebo group (increase of $10.7 \% p<0.05$ ). End power is a measurement that can be used to calculate critical power through critical power models (Vanhatalo et al., 2007). These results may be due central nervous system stimulation by caffeine and lowered sensitivity to pain which may be responsible for enhanced fatigue resistance. The study team found no differences in end-test power output between placebo and caffeinated groups. When compared to the placebo group, the mean power output for time intervals 0 seconds to 60, 90 and 120 seconds were all increased in the caffeinated group by $2.0 \%, 2.5 \%$, and $2.2 \%$ respectively. In all time intervals, the caffeinated group showed a mean power greater than the placebo group. In the placebo group, there was a faster decrease in average power output observed at 0,60 and 120 seconds, while the caffeine group was more resistant to fatigue and 
average power decreased at a slower pace. In the caffeinated group, ratings of perceived exertion did not change, but power output was increased, indicating caffeine intake could improve the tolerance to higher intensity exercises. Blood lactate concentration were elevated after exercise in both the caffeine and placebo group which was expected, however in the caffeinated group serum lactate post exercise $(11.25+/-2.58 \mathrm{mmol} / \mathrm{L})$ was significantly higher than in the placebo group $(9.80+/-1.76 \mathrm{mmol} / \mathrm{L})$. Plasma potassium levels were measured after the trials and one hour after ingestion of caffeine or the placebo, although both groups had increase in potassium levels, the caffeinated group $(3.87+/-0.22 \mathrm{mmol} / \mathrm{L})$ had a significantly lower potassium plasma concentration than the placebo group $(3.99+/-0.30 \mathrm{mmol} / \mathrm{L})$ after the $3 \mathrm{MT}$. Researchers in this study utilized the Shapiro-Wilk test to determine normality of the dependent variables, with a significance of $\mathrm{p}<0.05$. A $95 \%$ confidence interval and effect size were used to compare overall differences in the groups. A t-test was used to compare the means of caffeinated and placebo groups for $\mathrm{VO}_{2}$ max, heart rate and exercise outcomes. A within-participants repeated measures ANOVA was used to compare differences in serum lactate, sodium concentration, potassium concentration and $\mathrm{pH}$. If any changes were found to be statistically significant, a one-way ANOVA with Bonferroni post hoc comparisons were used to determine how much the differences between the two groups change over time.

A double-blind randomized crossover study conducted by Zbinden-Foncea et al. (2018) was done to determine the impact $5 \mathrm{mg}$ of caffeine per kilogram body mass had on performance of a countermovement jump (CMJ) by ten elite male volleyball players. Study subjects were selected if they had atleast six years of prior volleyball experience and trained at minimum 4 to 5 days a week or 14 hours in a week total for the past year. Testing was done over three separate occasions, during the first session individual caffeine and food intake were determined and 
participants were introduced to CMJ testing to ensure good technique. During the second session, subjects were randomized into a placebo or caffeinated groups with the third session being used as a crossover. Participants were given either $5 \mathrm{mg}$ per kilogram body mass anhydrous caffeine or a placebo in identical capsules to be ingested with $200 \mathrm{~mL}$ water, afterwards there was a one-hour period to allow for absorption. After ingestion subjects warmed up at $50 \mathrm{~W}$ on a stationary bike followed by additional three repetitions of $\mathrm{CMJ}$ at submaximal levels. CMJ involves two motions, the first being a downward eccentric countermovement following by a concentric upward jump. In between the two movements there was a slight pause to allow for power transfer.

Zbiden-Foncea et al. found the caffeinated group had a increase in flight time by $5.3 \%+/-$ $3.4 \%,(p<0.1)$ when compared to the placebo group. It was found the caffeine group had increased concentric phase peak force by $6.5 \% \pm 6.4 \% ;(p=.01)$ and peak power was increased by $16.2 \% \pm 8.3 \% ;(p<.01)$ in comparison to the placebo. Jump velocity and force produced during peak power were increased in the caffeinated group by $10.6 \% \pm 8.0 \%(\mathrm{p}<.01)$ and $6.0 \%$ $\pm 4.0 \%(\mathrm{p}<.01)$ respectively. Caffeine ingestion lead to an increase of $10.8 \% \pm 6.5 \% ;(\mathrm{p}<.01)$ in the height of the jump when compared to placebo. Specifically during the concentric phase, caffeine increased peak velocity $(12.6 \% \pm 7.4 \% ; \mathrm{p}<.01)$, peak power $(6.0 \% \pm 4.0 \% ; \mathrm{p}<.01)$, peak acceleration $(13.5 \% \pm 8.5 \% ; \mathrm{p}<.01)$, velocity at peak power $(10.6 \% \pm 8.0 \% ; \mathrm{p}<.01)$, peak displacement $(10.8 \% \pm 6.5 \% ; \mathrm{p}<.01)$ and force developed at peak power $(6.0 \% \pm 4.0 \% ; \mathrm{p}<.01)$ Other tested variable had no significant changes, but researchers found the caffeinated group had a greater elevation to diastolic blood pressure after the trials $(71.4 \pm 5.0$ before vs $81.2 \pm 11.3$ $\mathrm{mm} \mathrm{Hg} ; \mathrm{p}<.05)$. 
The study team used a Shapiro-Wilk test to determine normality of CMJ, followed by a paired-sample t-test to compare the mean and maximum jump heights of CMJ for the placebo and caffeine group. Afterwards, a two-way repeated-measures ANOVA with Bonferroni post hoc test were used to measure the difference between caffeine and placebo group for heart rate and blood pressure. The side effects of caffeine were measured using the McNemar nonparametric test and $\mathrm{p}<0.05$ was the level set for statistical significance.

A double-blind, randomized, crossover study conducted by Potgieter et al. (2018) was done to determine the effect caffeine had on triathlon performance in both males and female subjects. The study subjects consisted of fourteen male and twelve female registered triathlon athletes and were instructed to stop caffeine usage two weeks and limit exercise to 48 hours prior to testing. Trials consisted of two triathlons conducted two weeks apart, with each consisting of $1.5 \mathrm{~km}$ swimming portion, $40 \mathrm{~km}$ bike race and a $10 \mathrm{~km}$ run. Subjects were randomized into either the caffeinated or placebo group during the first test and then given the remaining treatment in the second. Both races were conducted at similar times of day with comparable weather conditions. Participants were given capsules containing $6 \mathrm{mg}$ of caffeine per kilogram of body pass or a placebo to be taken orally followed by an hour wait period for absorption. Average caffeine consumed was $652+/-72 \mathrm{mg}$ for males and $540+/-68 \mathrm{mg}$ for females. Researchers measured serum caffeine, leukocyte count, cortisol, testosterone, lactate and hematocrit before and after completion of the race and used the Borg Scale to determined subjects' rating of perceived exertion. A shortened profile of mood states (POMS) was used to determine mood state differences pre and post-triathlon in both groups.

Before initiation of the trials, average plasma caffeine levels were below $2 \mathrm{mg} / \mathrm{L}$ all subjects. After ingestion of the capsule, caffeine levels were maintained in the ergogenic range 
between 7 to $10 \mathrm{mg} / \mathrm{L}$ serum caffeine. Caffeine levels continued to rise through the triathlon due to the capsule used by the study group. The study findings demonstrate caffeine supplementation had a statistical significance improvement to overall performance in male subjects only, with a $1.7 \%$ reduction in time for males and $0.9 \%$ for females. Swimming time was shown to have the most reduction within the caffeinated group, with $4.5 \%$ and $2.8 \%$ reduction in males and females respectively. In $71 \%$ (10 of 14) of male subjects, caffeine supplementation improved the $10 \mathrm{~km}$ run portion of the triathlon, while $64 \%$ (9 of 14) of males had reduction to the $40 \mathrm{~km}$ cycling portion of the race. In female subjects, $67 \%$ (8 of 12) had an improvement to swim and cycling portions and $50 \%$ (6 of 12) demonstrated better running performance. No differences were observed in rating of perceived exertion or in POMS scores in the caffeinated and placebo groups. Both serum cortisol $(p<0.001)$ and testosterone $(p<0.001)$ levels were elevated, with cortisol levels being significantly greater in the caffeine group. Testosterone levels were found to be unrelated to caffeine ingestion, with the only differences found were between male and female subjects. Male subjects in the caffeinated group had statistically increased serum lactate levels $(\mathrm{p}<0.05)$ indicated caffeine slowed clearance of lactate. Leukocyte counts post-race were higher in caffeine group, with greater leukocytosis in male subjects. Analysis of the raw data in this study was done with ANOVA with restricted maximum likelihood (REML) to compare the caffeine and placebo groups. This allowed the researchers to compare an individual to their treatment group and gender as well as compare individual with themselves for placebo or caffeine ingestion. Maximum likelihood chi-squared test was utilized to compare non-qualitative data obtained by the study.

Fett et al. (2017) conducted a single-blind crossover study to determine the effect of caffeine on muscular strength and fatigue tolerance. The participants of study were eight women 
with ages ranging from 20 to 30 years old, with each having atleast one year of continuous resistance training. Average body mass index (BMI) for the subjects was restricted between 20 and $25 \mathrm{~kg} / \mathrm{m}^{2}$. During testing fifteen individuals were excluded from the results; eight did not perform any of the tests, two did not restrict themselves from caffeine and five had difficulty performing all the tests. After review of literature, the researchers decided to use $6 \mathrm{mg}$ per kilogram body mass of caffeine to ensure consistent ergogenic effects of caffeine which normally occur between 3 and 9 mg per kilogram body mass (Mora- Rodriguez, 2014). Both the caffeine and placebo groups were given identical capsules 30 minutes before testing began. The study was separated into four sessions done over four weeks. Each session consisted of four different movements, the first three tested muscular strength and consisted of a pull-down movement testing back and elbow flexor strength, hack squat to test lower limb strength and bench press to test chest, shoulder and elbow extensor strength. Every exercise was down three to five times to determine the one repetition maximum for each individual. To test caffeine's effects on muscle fatigue, a knee extension exercise was done for three sets in a drop set format of 100,80 and $60 \mathrm{~kg}$. Each set was done until exhaustion and total repetitions done were added together. The first session was used to determine baseline results for each individual with restriction from caffeine for 48 hours prior to the test. This session also allowed subjects to familiarize themselves with testing and the exercises. The second session subjects were given 6 mg per kilogram body mass caffeine with blinding before testing was conducted. The third session, subjects received the placebo and fourth session subjects again ingested caffeine. Each session had over 168 hours separating them to reduce the accumulation of fatigue and wear on the subjects. Researchers used analysis of variance (ANOVA) with a Tukey-Kramer Multiple comparison test to compare the means of each of the exercises for the final three sessions. 
Afterward, the mean of the two caffeine groups was compared to the means of the basal and placebo groups together, to examine the mean effects of caffeine. Delta was used to determine the level of effect caffeine had on the exercises, this was achieved by subtracting the mean of the two caffeine groups from the mean of the placebo and baseline groups. A paired t-test was then used to compare the delta values of each exercise.

Researchers found caffeine did not have an effect of the pull-down test, while it did show to increase hack squat strength in the second caffeine trial significantly $(\mathrm{p}<0.001)$. Bench press strength for the caffeine groups was elevated compared to the basal group $(\mathrm{p}<0.01$ and $\mathrm{p}<$ 0.001). Both caffeine groups had improvements to drop-set performance in relation to the placebo and basal groups $(\mathrm{p}<0.001$ and $\mathrm{p}<0.001)$. The mean of the caffeinated groups and placebo were shown to increase strength of the hack squat (mean of caffeine: $p<0.001$, placebo: $\mathrm{p}<0.05$ ) and bench press (mean of caffeine: $\mathrm{p}<0.001$, placebo: $\mathrm{p}<0.01$ ) when compared to the baseline. Resistance to exhaustion had a statistically significant increase for mean of the caffeine groups ( $\mathrm{p}<0.001)$ when compared to both the basal and placebo. Researchers found caffeine had a significant positive effect of the delta of the hack squat and bench press but no effect to the pull-down exercise.

\section{DISCUSSION}

After examination of the seven randomized controlled trials (Punete et al. 2017, Marques et al. 2018, Grgic et al. 2017, Cheng et al. 2016, Zbinden-Foncea et al. 2018, Potgieter et al. 2017, and Fett et al. 2017), this literature review sought to determine if caffeine was able to enhance athletic performance through physiological and neuromuscular effects. The end goal of this paper was to prove caffeine could be used as a performance enhancing agent prior to an athletic event to give athletes an advantage during competition. 
In the study conducted by Puente et al. (2017), researchers found caffeine was able to increase height reached in Abalakov jumps, amount of body impacts during a game, number of free throws attempted and made, total assists, total rebounds and offensive rebounds. A major finding in this study, not previously well researched was caffeine had a positive impact in overall basketball-specific performance. Caffeine did not demonstrate any effect on the accuracy of free throws, two-point or three-point shots during stimulated games indicating it only acts as ergogenic substance, but lacked net effects on sport-specific accuracy or skill-based actions. The increased physical performance in caffeinated group improved their positioning during stimulated games or allowing them to out-maneuver of their opponents putting them in more advantageous situations. This likely had a direct impact on number of assists, total and offensive rebounds during a game, which rely heavily on player coordination, position, and strength indicating caffeine could improve sport-specific skills. Rebounding was improved due to caffeine increasing jump height, which allowed players to reach the basketball before opponents. Another possible way caffeine may have improved sport-specific skills was by central nervous system stimulation through adenosine receptor antagonism. This may improve decision making and focus, which are essential during team sports. Lack of improvement to acceleration during CODAT testing contraindicates the findings of previous studies that show caffeine can improve acceleration. The researchers believe this may be due to CODAT testing multiple bouts of acceleration and deceleration, commonly performed in team-based sports while other studies had tested linear, continuous acceleration. The increase to total impacts in the caffeine group during the stimulated basketball games was an indicator caffeine influences player movement to be more physical. Increased physicality during basketball games allows players to use their strength to draw fouls and overpower opponents, giving themselves and their team an advantage. 
Caffeine may have also improved athletic performance by increasing self-perceived muscular strength and endurance, which may allow these athletes to performance at more intensive levels by avoiding symptoms of over-exertion and fatigue.

Negative effects of caffeine were centered insomnia due to the short six-hour window between the trials and bedtime. Other concerns the researchers had were possible under absorption of caffeine due to a pre-exercise meal. Possible confounding in this study could be caffeine's ergogenic effects relying on adequate glycogen stores in the liver and muscle which may not have been replenished. Limitations of the study were the experimental design of the games played. These did not accurately reflect real basketball games due to lack of substitutions and time-outs. Other limitations could be non-responders to caffeine could have shown no affect which might have interfered with the results of the study. Multiple trials were not conducted for both caffeine and non-caffeine groups putting too much emphasis on the two games played. Performance during team sports may have been enhanced or impaired by team cohesion and influence other players have on each other.

Marques et al. (2018) concluded caffeine did not improve times in overnight fasted athletes during an $800 \mathrm{~m}$ run. No differences were found in serum glucose, lactate and blood pressure between the caffeine and placebo groups. These results may be explained due to coffee, the mode of caffeine delivery in this study, might have contained various forms of caffeine such as caffeic acid and chlorogenic acid that may not be ergogenic. Previous studies conducted by Graham et al. (1998) found chlorogenic acid may interfere with the ergogenic activity of caffeine. Another possible explanation could be from theophylline found in coffee and decaffeinated coffee (placebo) which acts similar to caffeine, to antagonize adenosine receptors and stimulate carbohydrate oxidation to increase available ATP levels. The variable effects of 
caffeine within the study population may be due to polymorphisms in the CYP1A2 gene and ADORA2A adenosine receptor gene. Polymorphisms in the ADORA2A adenosine receptor may change the ability caffeine has to antagonize the adenosine receptor and could reduce its effects. CYP1A2 encodes for an enzyme of the p450 monooxygenase family involved in caffeine metabolism. CYP1A2 genotype AA are fast caffeine metabolizers and receive greater effects from caffeine than genotypes CC and AC. Genotype AA was found to have a $4.8 \%$ reduced time during a race with $2 \mathrm{mg}$ per kilogram body mass caffeine consumed verses genotype $\mathrm{CC}$ individuals that had a 13.7\% longer time to complete the run (Guest et al., 2018). The CYP1A2 gene was found to influence perceived exertion in individuals taking caffeine (Guest et al, 2018) but caffeine non-responders had no changes to perceived exertion rating. It was also found environmental temperature and humidity did not play a role in caffeine's ergogenic effects.

Possible limitations for this study were the small sample size of twelve participants, lack of repetition of the trials and lack of separation of responders and non-responders to caffeine which might have influenced the results. Prior to conducting the study, participants were not genotyped for their CYP1A2 and ADORA2A genes, therefore genetic differences in either might have led to lower mean values. Another limitation of this study might have been overnight fasting which could have depleted glycogen stores utilized by caffeine to produce its ergogenic effects.

A study conducted by Grgic et al. (2017) concluded caffeine ingestion enhanced lower body strength performance and reduce perceived exertion. No changes to upper body strength, endurance, pain perception or perceived exertion were found. Caffeine had an overall 2.8\% positive increase to lower body physical performance. A study conducted by Meur et al. (2009) found increases of atleast 3\% in performance may be enough to propel athletes past their 
competition and grant them victory. The improvements to lower body strength opposed findings of previous studies that found caffeine had no effect on lower body strength, these contrasting findings are likely explained by differences in the form caffeine was ingested, exercises performed or time for absorption. One way to measure the caffeine absorption is using salivary caffeine levels. Caffeine ingested as coffee or powder dissolved in liquid reaches ergogenic levels much quicker than caffeine ingested in capsular form. Other differences seen in the subjects could be due to differential response of an individual to caffeine. In caffeine responders, caffeine increased muscular strength by enhancing motor unit recruitment through central nervous stimulation. The only exercise without reduction in perceived exertion was the bench press due to its simple nature and being less physically taxing. The lack of changes in bench press strength may be due to the smaller muscles in the upper body having less motor unit recruitment after caffeine ingestion (Warren et al. 2010). The increases in muscular strength in the lower body was likely due to reduced perception of exertion allowing athletes to output more force and work. Caffeine influence on muscular endurance can reduce fatigue accumulation or reducing perception of fatigue, permitting athletes to perform the same exercises for longer periods.

The study design of this study could be a possible limitation due the bench press being performed at the end of each session. The accumulated fatigue and energy expenditure could have reduced the ability for athletes to perform the bench press and possibly limited their ability to perform as well. Another limitation the sample size of seventeen which might have impacted the results due to outliers in the subjects having a greater impact on the results than if a much greater sample size was utilized. The study was unable to determine how the placebo effect could 
affect physical performance because the study lacked a control or ensuring blinding was maintained.

Cheng et al. (2016) concluded the caffeine group had increased work above the end-test power (WEP), increased fatigue resistance and lowered serum potassium levels during 3MT. The researchers found the caffeine group had increased serum lactate without an effect on $\mathrm{pH}$ after 3MT. Caffeine ingestion was shown to have no effect on aerobic capacity due to no changes in end power (EP) of 3MT. EP can be used to measure critical power in an exercise which is predictive of aerobic capacities (Moritani et al. 1981). Researcher believe caffeine is able to increases WEP by reducing pain levels in late stages of 3MT exercise where anaerobic capacities are utilized. Reduction in pain perception was achieved through central nervous stimulation that decreases pain sensitivity, thereby allowing caffeinated individuals to have increased time for muscular activity before muscule pain and fatigue sets in and they must rest. WEP can be used to determine the curvature constant, a measurement of the abilities of an individual to perform actions to exhaustion. The accumulated oxygen deficit is the difference between the oxygen required by the body and oxygen taken up, this deficit can be widened by caffeine to enhance anaerobic capacity requiring less oxygen for the same actions. Caffeine was able to improve the muscle power of an athlete and slow the decline in power output by allowing athletes to exert more force while perceiving the same levels of exertion and be more tolerant of discomfort from exercise. Caffeine's adenosine receptor antagonism in the CNS can improve individual tolerance towards low and moderate intensity exercise by inducing hypoalgesia (Black et al. 2015). At higher intensities the caffeine-induced hypoalgesia was ineffective at opposing the excessive nociceptive activation. Studies found caffeine improved muscle strength by increasing motor unit activation and the total force produced during contraction of muscles (Bazzucchi, 2011). 
Caffeine use was thought to be effective in sports due to the majority of actions and movements performed are at submaximal levels. The caffeinated group was found to have lower interstitial potassium levels than placebo which may enhance performance by maintaining membrane potential and permitting rapid and continuous muscular stimulation. These effects are achieved through caffeine's enhancement of the sodium potassium ATPase pump activity.

Limitations for this study were the small sample size of fifteen and lack of control group. Other limitations could be the relationship between serum potassium and interstitial muscle potassium levels due to caffeine showing no changes to serum potassium levels during in the study.

Zbinden-Foncea et al. (2018) determined 5mg per kilogram body mass caffeine ingested by volleyball athletes led to increased jump height, velocity of jump and force produced during jump in countermovement-jump (CMJ) performance. The caffeine group had increased maximum velocity, maximum acceleration, maximum power, and maximum force generated during concentric contraction. These results mirrored those of Del Coso et al. (2014), who found caffeine increased height and power of CMJ by $5 \%$ and $2.5 \%$. The effects achieved by caffeine ingestion in athletes unfamiliar to $\mathrm{CMJ}$ mimic the results of athletes with previous $\mathrm{CMJ}$ training (Del Coso et al. 2014). Zbinden-Focea et al. (2018) determined caffeine did not affect jump mechanics with similar results being found in athletes of other sports, such as soccer and basketball players when they were tested with CMJ. The study team believed the ergogenic effects of caffeine were explained by its central stimulation of the nervous system through adenosine receptor antagonism. Adenosine has inhibitory action of neural excitability and synaptic transmission. Caffeine improved intermuscular and intramuscular coordination by increasing overall motor unit recruitment and led to elevated calcium release from the myocyte 
sarcoplasmic reticulum leading to increased myocyte contractility. Researchers found single nucleotide polymorphisms within intron 1 of CYP1A2 enhanced its breakdown of caffeine which blunted caffeine's ergogenic effects in individuals with this mutation. This served as a major limitation of the study due to lack of separation of participants based of their CYP1A2 genotype. Another limitation was the small sample size of ten and the lack of placebo which may lead to confounding from the placebo effect. Lack of repetition of trials is another limitation, due to outliers having a more profound effect on the data.

Potgier et al. (2018) concluded usage of caffeine enhanced triathlon performance in almost all individuals tested. Males subjects improved by $1.7 \%$ while females subject by $0.9 \%$. Improvement were less pronounced in this study compared to others due to the realistic nature of the trials and participants actively competing against each other. Variations in caffeine habituation between the genders was likely explained by females consuming caffeine on average at $501 \pm 649 \mathrm{mg}$ per day than males at $337 \pm 345 \mathrm{mg}$ per day (Ganio et al., 2009). The researchers believed caffeine ingestion led to increased glucose levels, changes in serum ion levels and decreased perceived exertion ultimately causing its ergogenic effects. Caffeine might have been especially beneficial for low to moderate intensity exercise by boosting force production of skeletal muscles. Caffeine might have been less effective for intense exercise because the majority of motor units were already in use and caffeine had no direct effect on muscle. This might have led to swimming timings to decrease by $3.7 \%$ on average while overall time on decreased by $1.3 \%$ due to the moderate intensity of swimming. The presence on nonresponders and responders in the study group functioned as a limitation to the study due to lack of stratification of the groups. Sport-specific training and adaptation serve as other limiting factors in the study due to top-forming participants benefiting less from the ergogenic effects of 
caffeine than lower performing individuals. The central effects of caffeine allowed athletes to separate perceived effort during physical activity from the symptoms of physical activity such as pain and fatigue and allow them to exert higher levels of effort. Another source of limitation in this study was the differences in males and females exercise-induced cortisol levels which may change overall bodily response to exercise. Sample size, lack of control and lack of trial repetition were other sources of limitation.

The final study analysed for this literature review was performed by Fett et al. (2017) and concluded caffeine improved strength of the lower limbs and increased tolerance to fatigue. The research team believed caffeine's central nervous stimulation increased muscular contraction and improve total output by reducing experience of fatigue. Caffeine was thought to be beneficial in movements requiring recruitment of large muscle groups because it can reduce CNS fatigue that occurs through excessive muscular stimulation. Due to caffeine use, muscles were able contract more often and at higher intensities, which was demonstrated by an increase of $1 \mathrm{~kg}$ and $2 \mathrm{~kg}$ for the hack squat in the caffeinated trials when compared to the placebo group. The second caffeine trial had improvements in the drop set exercise and bench press while the first trial lacked improvement which may point to a learning period the body must go through to work efficiently with caffeine supplementation. The negative effects of caffeine included gastrointestinal distress, anxiety and insomnia and were seen in a few of the subjects. Those who infrequently ingested caffeine experienced more symptoms and at higher levels. Limitations of this study were its small sample size and the absence of measurement to changes in glucose, lactate and other physiological variables before and after the exercises were performed.

The studies analyzed had many sources of limitation, primarily all of them had small sample sizes with many lacking control groups. There was lack of repetition of trials in both 
placebo and caffeinated group which would have accounted for any gradual improvements in the trials from experience. No stratification was done to separate caffeine responders and nonresponders, and none of the studies considered the effects of polymorphism in the CYP1A2 and ADORA2A genes.

\section{Future Directions and Conclusion}

Possible avenues for future research would be quantifying the effect each genotype of the CYP1A2 gene would have on the ergogenic effects of caffeine and determining the ideal dosing to achieve ergogenic activity while avoiding side effects. More research should be done with greater sample sizes and additional repetition of trials. Caffeine research should also be done separating caffeine responders and non-responders as well as research for its use during training for athletic competition. There also should be research conducted on the ideal timing for caffeine ingestion to ensure ergogenic activity was reached and maintained through competition.

After the analysis of the seven primary articles, six out of the seven found caffeine to have a statistically significant impact on improving the physical performance of athletes. Two of the studies tested caffeine in a realistic sporting setting, and found a statistically significantly improvement in both for sport-specific performance. Based on these findings, caffeine can be used prior to competitions by athletes to utilize its ergogenic properties to boost athletic performance.

\section{ACKNOWLEDGEMENTS}

The author declares there were no conflicts of interest or funding in regard to this paper. 


\section{REFERENCES}

Bazzucchi, I., Felici, F., Montini, M., Figura, F., \& Sacchetti, M. (2011). Caffeine improves neuromuscular function during maximal dynamic exercise. Muscle \& nerve, 43(6), 839844. https://doi.org/10.1002/mus.21995

Black, C. D., Waddell, D. E., \& Gonglach, A. R. (2015). Caffeine's Ergogenic Effects on Cycling: Neuromuscular and Perceptual Factors. Medicine and science in sports and exercise, 47(6), 1145-1158.

Borg G. (1970). Perceived exertion as an indicator of somatic stress. Scandinavian journal of rehabilitation medicine, 2(2), 92-98.

Brzycki, M (1993). Strength Testing-Predicting a One-Rep Max from Reps-to-Fatigue. Journal of Physical Education, 64(1), 88-90, doi: 10.1080/07303084.1993.10606684

Cheng, C., Hsu, W., Kuo, Y., Shih, M., \& Lee, C. (2016). Caffeine ingestion improves power output decrement during 3-min all-out exercise. European Journal of Applied Physiology, 116(9), 1693-1702. doi:10.1007/s00421-016-3423-x

Del Coso, J., Pérez-López, A., Abian-Vicen, J., Salinero, J. J., Lara, B., \& Valadés, D. (2014). Enhancing physical performance in male volleyball players with a caffeinecontaining energy drink. International journal of sports physiology and performance, 9(6), 1013-1018. https://doi.org/10.1123/ijspp.2013-0448

Fett, C. A., Aquino, N. M., Schantz Junior, J., Brandão, C. F., de Araújo Cavalcanti, J. D., \& Fett, W. C. (2018). Performance of muscle strength and fatigue tolerance in young trained women supplemented with caffeine. The Journal of sports medicine and physical fitness, 58(3), 249-255. https://doi.org/10.23736/S0022-4707.17.06615-4 
Ganio, M. S., Klau, J. F., Casa, D. J., Armstrong, L. E., \& Maresh, C. M. (2009). Effect of caffeine on sport-specific endurance performance: a systematic review. Journal of strength and conditioning research, 23(1), 315-324.

Graham, T. E., Hibbert, E., \& Sathasivam, P. (1998). Metabolic and exercise endurance effects of coffee and caffeine ingestion. Journal of applied physiology (Bethesda, Md. : 1985), 85(3), 883-889. https://doi.org/10.1152/jappl.1998.85.3.883

Grgic, J., \& Mikulic, P. (2017). Caffeine ingestion acutely enhances muscular strength and power but not muscular endurance in resistance-trained men. European Journal of Sport Science, 17(8), 1029-1036. doi:10.1080/17461391.2017.1330362

Guest, N., Corey, P., Vescovi, J., \& El-Sohemy, A. (2018). Caffeine, CYP1A2 Genotype, and Endurance Performance in Athletes. Medicine and science in sports and exercise, 50(8), 1570-1578. https://doi.org/10.1249/MSS.0000000000001596

Marques, A., Jesus, A., Giglio, B., Marini, A., Lobo, P., Mota, J., \& Pimentel, G. (2018). Acute Caffeinated Coffee Consumption Does not Improve Time Trial Performance in an 800-m Run: A Randomized, Double-Blind, Crossover, Placebo-Controlled Study. Nutrients, 10(6), 657. doi:10.3390/nu10060657

Moher, D., Liberati, A., Tetzlaff, J., Altman, D. G., \& PRISMA Group (2009). Preferred reporting items for systematic reviews and meta-analyses: the PRISMA statement. PLoS medicine, 6(7), e1000097. https://doi.org/10.1371/journal.pmed.1000097

Mora-Rodriguez, R., \& Pallarés, J. G. (2014). Performance outcomes and unwanted side effects associated with energy drinks. Nutrition reviews, 72 Suppl 1, 108-120. https://doi.org/10.1111/nure.12132

Moritani, T., Nagata, A., deVries, H. A., \& Muro, M. (1981). Critical power as a measure of 
physical work capacity and anaerobic threshold. Ergonomics, 24(5), 339-350.

\section{https://doi.org/10.1080/00140138108924856}

Pickering, C., \& Kiely, J. (2018). Are the Current Guidelines on Caffeine Use in Sport Optimal for Everyone? Inter-individual Variation in Caffeine Ergogenicity, and a Move Towards Personalised Sports Nutrition. Sports medicine (Auckland, N.Z.), 48(1), 7-16. https://doi.org/10.1007/s40279-017-0776-1

Potgieter, S., Wright, H. H., \& Smith, C. (2018). Caffeine Improves Triathlon Performance: A Field Study in Males and Females. International Journal of Sport Nutrition and Exercise Metabolism, 28(3), 228-237. doi:10.1123/ijsnem.2017-0165

Puente, C., Abián-Vicén, J., Salinero, J., Lara, B., Areces, F., \& Coso, J. D. (2017). Caffeine Improves Basketball Performance in Experienced Basketball Players. Nutrients, 9(9), 1033. doi:10.3390/nu9091033

Urry, E., \& Landolt, H. (2014). Adenosine, Caffeine, and Performance: From Cognitive Neuroscience of Sleep to Sleep Pharmacogenetics. Sleep, Neuronal Plasticity and Brain Function Current Topics in Behavioral Neurosciences, 331-366. doi:10.1007/7854_2014_274

Vanderveen, J., Armstrong, L., Butterfield, G., Chenoweth, W., Dwyer, J., J., Fernstrom \& Kanarek, R. (2001). Caffeine for the sustainment of mental task performance: Formulations for military operations. Washington, D.C.: National Academy Press.

Vanhatalo, A., Doust, J. H., \& Burnley, M. (2007). Determination of critical power using a 3min all-out cycling test. Medicine and science in sports and exercise, 39(3), 548-555. https://doi.org/10.1249/mss.0b013e31802dd3e6

Zbinden-Foncea, H., Rada, I., Gomez, J., Kokaly, M., Stellingwerff, T., Deldicque, L., \& 
Peñailillo, L. (2018). Effects of Caffeine on Countermovement-Jump Performance Variables in Elite Male Volleyball Players. International Journal of Sports Physiology and Performance, 13(2), 145-150. doi:10.1123/ijspp.2016-0705 


\section{APPENDIX A}

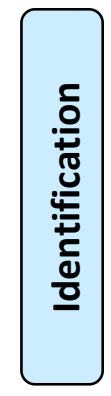

\section{Records identified through} database searching $(n=46)$

Additional records identified through other sources $(\mathrm{n}=1)$
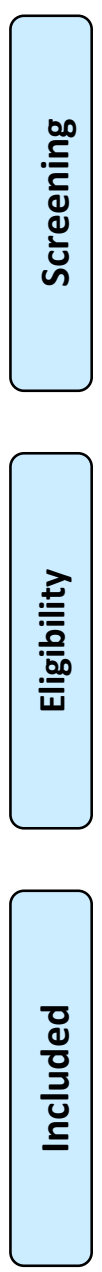

Excluded articles $(\mathrm{n}=40)$

- Solely neurological $(n=5)$

- Multiple variables tested $(\mathrm{n}=15)$

- Study parameters too specific $(n=6)$

- Caffeine given in energy drink form $(n=8)$

- Methods to measurement of the effects of caffeine too nonspecific $(n=6)$

Figure 1. PRISMA (Moher, 2009) flow diagram displaying exclusion and selection of articles 


\section{APPENDIX B}

Table 1. Evidence table of primary sources used in results

\begin{tabular}{|c|c|c|c|c|c|c|}
\hline First Author & Date of publication & $\begin{array}{l}\text { Study } \\
\text { Design }\end{array}$ & $\begin{array}{l}\text { Level } \\
\text { of } \\
\text { Evide } \\
\text { nce }\end{array}$ & Study Population & Therapy/Exposure & Outcome/results \\
\hline $\begin{array}{l}\text { 1. Puente, } \\
\text { Carlos }\end{array}$ & June 2017 & RCT & 1 & $\begin{array}{l}\text { Twenty professional and semi- } \\
\text { professional male and female } \\
\text { basketball players }\end{array}$ & $\begin{array}{l}3 \mathrm{mg} / \mathrm{kg} \text { body } \\
\text { mass } \\
\text { anhydrous } \\
\text { caffeine or a } \\
\text { placebo }\end{array}$ & $\begin{array}{l}\text { Caffeine } \\
\text { supplementation } \\
\text { increased average } \\
\text { Abalakov jump } \\
\text { height, number of } \\
\text { body impacts, and } \\
\text { basketball } \\
\text { performance } \\
\text { index rating }\end{array}$ \\
\hline $\begin{array}{l}\text { 2. Marques, } \\
\text { Alexandre C. }\end{array}$ & April 2018 & $\mathrm{RCT}$ & 1 & $\begin{array}{l}\text { Twelve males with amateur } \\
\text { running experience that had } \\
\text { fasted overnight ( } 8-12 \text { hours) }\end{array}$ & \begin{tabular}{|l|}
$5.5 \mathrm{mg} / \mathrm{kg}$ \\
caffeine \\
ingested as \\
coffee or a \\
decaffeinated \\
coffee placebo \\
\\
\end{tabular} & $\begin{array}{l}\text { No differences } \\
\text { were found } \\
\text { between the } \\
\text { caffeine and } \\
\text { placebo group in } \\
\text { time to complete } \\
\text { the } 800 \mathrm{~m} \text { race and } \\
\text { no changes in } \\
\text { rating of } \\
\text { perceived } \\
\text { exertion, blood } \\
\text { glucose or blood } \\
\text { lactate levels }\end{array}$ \\
\hline 3. Grgic, Jozo & May 2017 & $\mathrm{RCT}$ & 1 & $\begin{array}{l}\text { Seventeen male participants with } \\
\text { over one year of resistance } \\
\text { training and had been training } \\
\text { atleast } 3 \text { time a week for the last } \\
\text { six months }\end{array}$ & \begin{tabular}{|l|} 
Caffeine \\
supplementati \\
on $6 \mathrm{mg} / \mathrm{kg}$ \\
body mass or \\
a placebo one \\
hour before \\
testing
\end{tabular} & $\begin{array}{l}\text { The caffeinated } \\
\text { group had } \\
\text { increased one } \\
\text { repetition } \\
\text { maximum for } \\
\text { back squat } \\
\text { strength, } \\
\text { increased power } \\
\text { in the seated } \\
\text { medicine ball } \\
\end{array}$ \\
\hline
\end{tabular}




\begin{tabular}{|c|c|c|c|c|c|c|}
\hline First Author & Date of publication & $\begin{array}{l}\text { Study } \\
\text { Design }\end{array}$ & $\begin{array}{l}\text { Level } \\
\text { of } \\
\text { Evide } \\
\text { nce }\end{array}$ & Study Population & Therapy/Exposure & Outcome/results \\
\hline & & & & & & $\begin{array}{l}\text { throw, increased } \\
\text { overall lower } \\
\text { body strength, } \\
\text { and reduction in } \\
\text { perceived } \\
\text { exertion and pain } \\
\text { perception }\end{array}$ \\
\hline $\begin{array}{l}\text { 4. Cheng, } \\
\text { Ching-Feng }\end{array}$ & January 2016 & $\mathrm{RCT}$ & 1 & $\begin{array}{l}\text { Fifteen Division } 1 \text { college male } \\
\text { basketball players }\end{array}$ & $\begin{array}{l}6 \mathrm{mg} / \mathrm{kg} \text { body } \\
\text { mass of either } \\
\text { caffeine or a } \\
\text { placebo }\end{array}$ & $\begin{array}{l}\text { It was found } \\
\text { caffeine increased } \\
\text { work above end- } \\
\text { power (WEP) and } \\
\text { power output } \\
\text { between } 60 \text { to } 150 \\
\text { seconds }\end{array}$ \\
\hline $\begin{array}{l}\text { 5. Zbiden- } \\
\text { Foncea, } \\
\text { Hermann }\end{array}$ & May 2017 & RCT & 1 & $\begin{array}{l}\text { Ten male volleyball players from } \\
\text { the Chilean national team }\end{array}$ & $\begin{array}{l}5 \mathrm{mg} / \mathrm{kg} \\
\text { anhydrous } \\
\text { caffeine or a } \\
\text { placebo }\end{array}$ & $\begin{array}{l}\text { Caffeine intake } \\
\text { increased } \\
\text { countermovement } \\
\text {-jump peak } \\
\text { concentric force, } \\
\text { peak power and } \\
\text { increased the } \\
\text { maximum height, } \\
\text { velocity, power } \\
\text { and acceleration } \\
\text { achieved during } \\
\text { the jump } \\
\end{array}$ \\
\hline $\begin{array}{l}\text { 6. Potgieter, } \\
\text { Sunita }\end{array}$ & $\begin{array}{l}\text { Decemeber } \\
2017\end{array}$ & $\mathrm{RCT}$ & 1 & $\begin{array}{l}\text { Twenty-six male and female } \\
\text { triathlon athletes }\end{array}$ & $\begin{array}{l}6 \mathrm{mg} / \mathrm{kg} \text { body } \\
\text { mass caffeine } \\
\text { or a placebo }\end{array}$ & $\begin{array}{l}\text { Caffeine led to a } \\
\text { mean } 3.7 \% \\
\text { decrease in } \\
\text { swimming times } \\
\text { and mean } 1.3 \% \\
\text { decrease in total } \\
\text { time to complete } \\
\text { the triathlon. }\end{array}$ \\
\hline 7. Fett, Carlos & April 2017 & $\mathrm{RCT}$ & 1 & $\begin{array}{l}\text { Eight women between ages } 20 \text { to } \\
30 \text { years old and BMI between } \\
20-25 \text { with atleast one year of } \\
\text { continuous resistance training }\end{array}$ & $\begin{array}{l}6 \mathrm{mg} / \mathrm{kg} \text { body } \\
\text { mass of } \\
\text { caffeine or a } \\
\text { placebo } 30 \\
\text { minutes } \\
\text { before }\end{array}$ & $\begin{array}{l}\text { Caffeine } \\
\text { increased hack } \\
\text { squat strength and } \\
\text { for the second } \\
\text { caffeine trial, } \\
\text { there was an }\end{array}$ \\
\hline
\end{tabular}




\begin{tabular}{|c|c|c|c|c|c|}
\hline First Author & Date of publication & $\begin{array}{l}\text { Study } \\
\text { Design }\end{array}$ & $\begin{array}{l}\text { Level Study Population } \\
\text { of } \\
\text { Evide } \\
\text { nce }\end{array}$ & Therapy/Exposure & Outcome/results \\
\hline & & & & $\begin{array}{l}\text { performance } \\
\text { tests }\end{array}$ & $\begin{array}{l}\text { increase to bench } \\
\text { press strength, } \\
\text { and increased } \\
\text { time until } \\
\text { exhaustion }\end{array}$ \\
\hline
\end{tabular}

